\title{
Genome-scale model reconstruction of the methylotrophic yeast Ogataea polymorpha
}

\author{
Ulf W Liebal ${ }^{1}$, Brigida A Fabry', Aarthi Ravikrishnan² , Constantin VL Schedel ${ }^{1}$, Simone Schmitz ${ }^{1}$, \\ Lars M Blank ${ }^{1 *}$ and Birgitta E Ebert ${ }^{1,3,4}$
}

\begin{abstract}
Background: Ogataea polymorpha is a thermotolerant, methylotrophic yeast with significant industrial applications. While previously mainly used for protein synthesis, it also holds promise for producing platform chemicals. O. polymorpha has the distinct advantage of using methanol as a substrate, which could be potentially derived from carbon capture and utilization streams. Full development of the organism into a production strain and estimation of the metabolic capabilities require additional strain design, guided by metabolic modeling with a genome-scale metabolic model. However, to date, no genome-scale metabolic model is available for O. polymorpha.
\end{abstract}

Results: To overcome this limitation, we used a published reconstruction of the closely related yeast Komagataella phaffii as a reference and corrected reactions based on KEGG and MGOB annotation. Additionally, we conducted phenotype microarray experiments to test the suitability of 190 substrates as carbon sources. Over three-quarter of the substrate use was correctly reproduced by the model and 27 new substrates were added, that were not present in the K. phaffii reference model.

Conclusion: The developed genome-scale metabolic model of O. polymorpha will support the engineering of synthetic metabolic capabilities and enable the optimization of production processes, thereby supporting a sustainable future methanol economy.

Keywords: Biotechnology, Genome-scale metabolic model, Metabolic reconstruction, Metabolic engineering, COBRA, Methylotrophy

\section{Background}

Ogataea polymorpha (Hansenula polymorpha; Pichia angusta) is a widely used yeast for biotechnological applications. It is environmentally ubiquitous and has been isolated, among others, from orange juice, maize meal, and insect guts [1]. The ability to metabolize methanol stems from its close association to the phyllosphere [2]. $O$.

*Correspondence: lars.blank@rwth-aachen.de

${ }^{1}$ Institute of Applied Microbiology-iAMB, Aachen Biology and

Biotechnology-ABBt, RWTH Aachen University, Worringer Weg 1, 52074

Aachen, Germany

Full list of author information is available at the end of the article polymorpha has some unique features among methylotrophic yeasts such as high growth rate, temperature tolerance, and nitrate fixation [3] and has been optimized to express peptides and proteins [4]. A particular benefit for biotechnological applications is the lack of byproducts even on high glucose feeds $[4,5]$. Furthermore, the conversion of methanol to succinic acid via methylotrophic yeasts was found to provide a competitive alternative to conventional petrochemical approaches in a computational comparison of various microbial carbon fixation strategies [6].

Methylotrophy in yeast is restricted to a single multigenus clade, with its most prominent members being

(c) The Author(s). 2021, corrected publication 2021 Open Access This article is licensed under a Creative Commons Attribution 4.0 International License, which permits use, sharing, adaptation, distribution and reproduction in any medium or format, as long as you give appropriate credit to the original author(s) and the source, provide a link to the Creative Commons licence, and indicate if changes were made. The images or other third party material in this article are included in the article's Creative Commons licence, unless indicated otherwise in a credit line to the material. If material is not included in the article's Creative Commons licence and your intended use is not permitted by statutory regulation or exceeds the permitted use, you will need to obtain permission directly from the copyright holder. To view a copy of this licence, visit http://creativecommons.org/licenses/ by/4.0/. The Creative Commons Public Domain Dedication waiver (http://creativecommons.org/publicdomain/zero/1.0/) applies to the data made available in this article, unless otherwise stated in a credit line to the data. 
Komagataella phaffii, O. polymorpha, and Candida arabinofermentans [7]. The phylogeny of methylotrophic yeast is complex and subject to recent updates [8]. The Ogataea strains most frequently mentioned in the literature are DL-1 (ATCC 26012), CBS4732 (ATCC 34438, NRRL-Y-5445, TB-3) and NCYC 495 (ATCC 14754, NRRL Y-1798) $[1,9,10]$. The genomes of all three strains have been sequenced and annotated $[1,7,10]$. A genomic comparison of O. polymorpha DL-1 and $K$. phaffii showed that they share three-quarters of enzymes whereas the non-overlapping proteome contains mostly hypothetical, uncharacterized proteins, indicating their close relationship and overlapping metabolic features [10].

The methylotrophic model organism $K$. phaffii is one of the few yeasts that have the ability to use methanol as the only carbon source for energy production. K. phaffii was developed into an effective producer of recombinant proteins based on the strength of the native methanolresponsive promoter expression system. The wealth of knowledge has lead to the development of metabolic models on the genome level (GSMM) for simulation and strain engineering $[11,12]$. The most recent models are iMT1026v3 and iRY1243 [13, 14]. Based on the close relationship among methylotrophic yeasts and the availability of sequencing data in genomic databases, the development of a GSMM for the metabolism of O. polymorpha is desirable.

$O$. polymorpha and $K$. phaffii share many similarities especially regarding their methanol metabolism. However, several metabolic features are distinctive. $O$. polymorpha is capable of growth at $50^{\circ} \mathrm{C}$ and is therefore one of the most thermotolerant eukaryotic microorganisms [15]. In contrast to K. phaffii, O. polymorpha can ferment xylose to ethanol [16] and can assimilate nitrate [17]. Moreover, high activity of the AOX promoter with glycerol as the sole carbon source or under glucose starvation is a unique feature of O. polymorpha. In K. phaffii, activity of AOX strictly depends on the presence of methanol [18].

Here, we present iUL909 as the first GSMM of the biotechnological relevant methylotrophic yeast $O$. polymorpha NCYC 495. The model is based on existing models for $K$. phaffii, extended by species-specific substrate utilization identified in phenotype microarrays. Model predictions of growth rates were found to represent experimental growth in different conditions. We tested the performance for overproduction of lactate, and succinate with methanol and glucose as substrates for biotechnological applications and identified potential targets for amplification of reaction activities.

\section{Results and discussion}

Here, we report on the construction of a genomescale metabolic model of the methylotrophic yeast $O$. polymorpha named iUL909. iUL909 was generated on the basis of an existing metabolic model of $K$. phaffii iMT1026v3 [13, 14]. Gene identifiers from $K$. phaffii in iMT1026v3 were replaced by those of homologs from O. polymorpha. The model was validated against physiological data from substrate utilization tests with phenotype microarrays and shake flask experiments, as well as growth rates from the literature. We further simulated the production of industrially relevant molecules to identify future biotechnological applications. The validity of the SBML model was tested with Memote [19].

\section{General properties of iUL909}

The close relationship among methylotrophic yeasts is reflected by a high overlap of homologs between $O$. polymorpha and K. phaffii [7]. The details of our reconstruction and a comparison with the reference GSMMs are shown in Fig. 1 and Table 1. We mapped genes from K. phaffii to O. polymorpha using a homolog-search (see Material and Methods section) which failed to find 84 genes (see Additional file 3). However, we added 114 new reactions and 39 new genes (see Additional file 3) from two sources: (i) additionally annotated GPRs from the iRY1243 GSMM, and (ii) genes identified for reactions required for the metabolization of carbon substrates identified with the Biolog ${ }^{\odot}$ Phenotype microarray. iRY1243 differs from the reference model iMT1026v3 only in reactions associated with transcription/translation, signaling and protein turnover. Since iUL909 covers only metabolic reactions it was not amended with these additional reactions from iRY1243. Overall, the common origin and high overlap of the three GSMMs is visible from the comparable number of the compartmentalized reactions (Fig. 1).

\section{Comparison of iUL909 with iMT1026}

The O. polymorpha GSMM reproduces experimental growth rates with growth parameters from iMT1026. All growth parameters were taken from the reference model iMT1026v3, namely growth- and non-growthassociated maintenance energy (GAM, NGAM) as well as the biomass reaction [13]. The substrates tested were methanol, glucose, and glycerol, industrially relevant sources for which literature values of growth rate and substrate uptake rate are available [5, 20-22]. The Pearson correlation coefficient of 0.9996 between predicted and experimental growth rate supports using GAM, NGAM and biomass equation from iMT1026v3 (Fig. 2). In particular, the growth characteristics on methanol reported by Van Dijken et al. [20] for O. polymorpha (Fig. 2, circles) is reproduced, underscoring the similarity of biomass composition and methanol metabolism between $O$. polymorpha and $K$. phaffii.

Notwithstanding the high degree of similarity between $K$. phaffii and $O$. polymorpha there were 84 genes in iMT1026 without a detected homolog in O. polymorpha. 

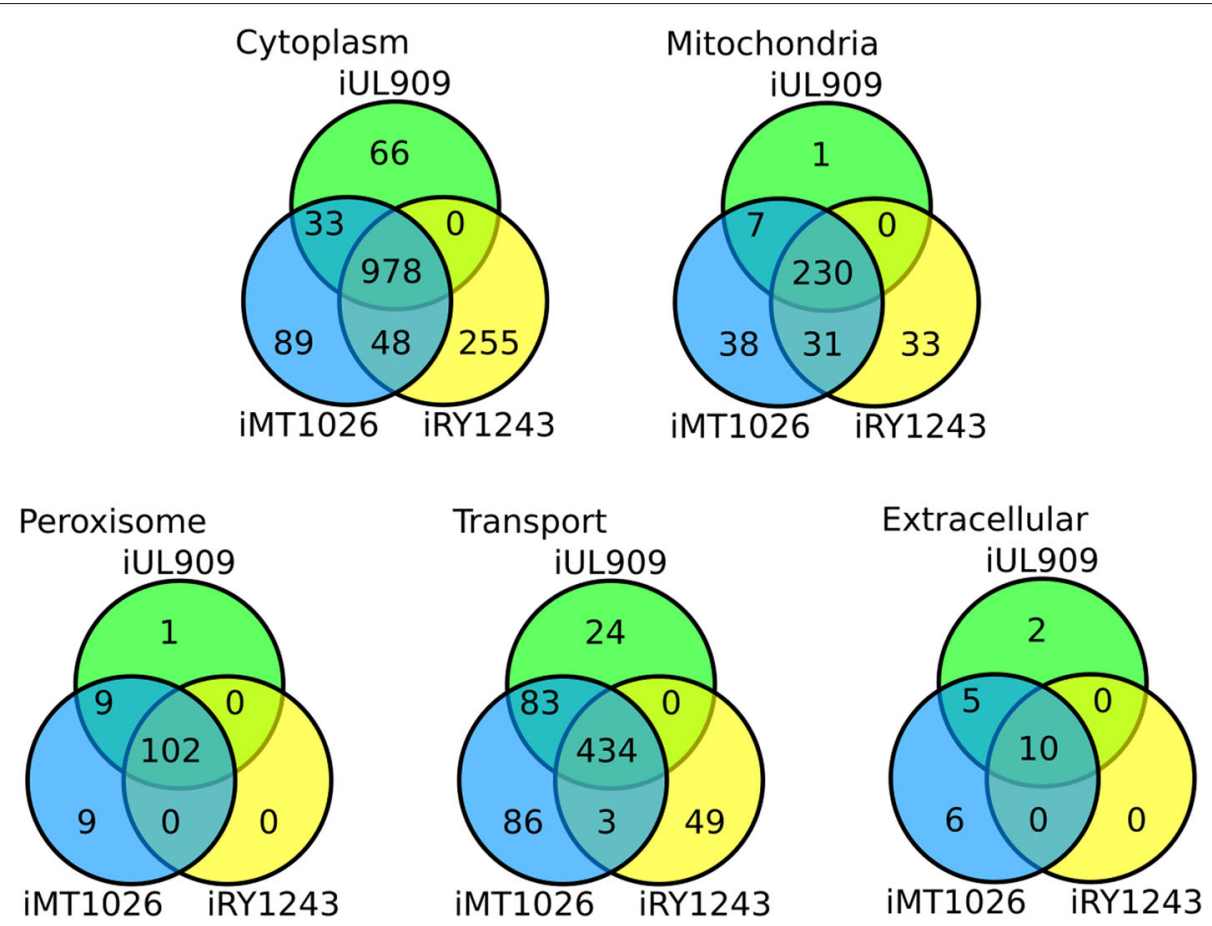

Fig. 1 Venn diagrams of compartment specific reactions overlap. The genome-scale metabolic model (GSMM) of K. phaffii iMT1026 [13] was used as a reference to construct the GSMM for O. polymorpha because of metabolic and phylogenetic similarities (see text). iMT1026 was further adapted for biotechnology in the GSMM iRY1243 [14]. The reactions are fully overlapping for the compartments Golgi, nucleus, endoplasmatic reticulum and vacuole

Because many reactions were catalyzed by alternative enzymes, the gene-protein-reaction relationships (GPR) was still satisfied for 62 genes, but 22 gene products were sole catalysts for the associated reactions (see Additional file 3). These 22 reactions lacked a GPR, caused by gene function replacement or loss. Replacement is likely when upstream and downstream reactions are annotated, loss (or misannotation in iMT1026) is likely for orphan reactions without connections to the metabolism. Among those orphan reactions, nine were deleted because they could not carry flux. For example, a potential misannotation was identified for $4 \alpha$-hydroxytetrahydrobiopterin dehydratase (EC number 4.2.1.96) in iMT1026 for which neither KEGG nor the model itself would allow metabolite flux. However, thirteen reactions were retained because they were metabolically well connected, and their deletion would result in reaction gaps. For exam- ple, the identities and the location of all non-proton pumping mitochondrial NADH dehydrogenase system is currently not sufficiently well known. We decided to retain dehydrogenase reactions from the reference model iMT1026. More experiments and data will guide improvements regarding the actual electron and proton homeostasis.

\section{Substrate identification with biolog plates}

Substrate tests with phenotype-microarray plates were conducted to compare predictions of the reference GSMM of $K$. phaffii iMT1026 with the actual growth phenotype of $O$. polymorpha. Biolog's Phenotype Microarray $^{\mathrm{Tm}}$ plates test was used to analyze the metabolic utilization of 190 carbon substrates (see Material and Methods section). Table 2 shows the overlap and differences between experiment and simulation run with

Table 1 Feature comparison of the O. polymorpha GSMM with respect to the reference GSMM of K. phaffii

\begin{tabular}{llll}
\hline & iUL909 & iMT1026v3 & iRY1243 \\
\hline Genes & 909 & 1026 & 1243 \\
Metabolites & 1639 & 1706 & 1740 \\
Reactions & 2263 & 2237 & 2407 \\
\hline
\end{tabular}




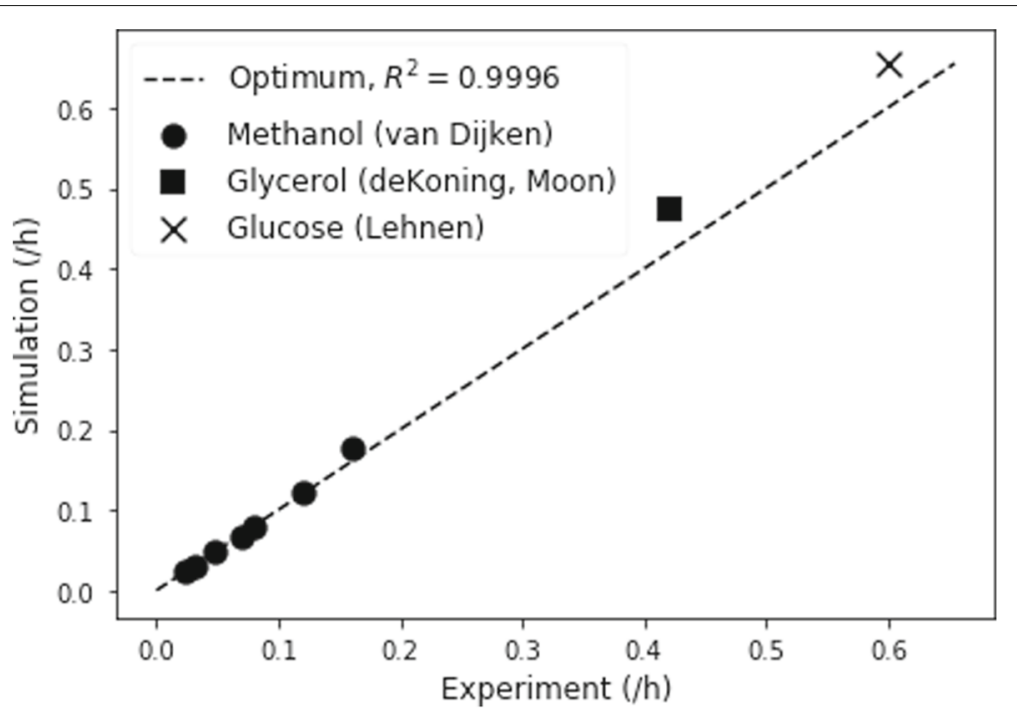

Fig. 2 Comparison of experimental and simulated growth rates. Comparison of experimentally measured and simulated growth rates on methanol, glucose, and glycerol. Experimental data were taken from literature [5, 20-22]. Biomass composition, growth-, and non-growth associated maintenance (GAM, NGAM) are based on [13] for the respective substrate

the $K$. phaffii GSMM iMT1026. Correct growth phenotypes were predicted in $77 \%$ cases (13 positives and 134 negatives). In eight cases no growth was experimentally measured, whereas it was predicted by the simulation. Substrates of this class comprised mainly organic acids associated with the TCA cycle (see Additional file 2). We tested growth separately in shake flask experiments and observed growth after two days for all of the eight substrates (Additional file 2). We hypothesize, that the base medium of the Biolog Phenotype Microarray $^{\text {тM }}$ was inappropriate for supporting growth with the eight substrates and/or that the cultivation time was too short for achieving adaptation of O. polymorpha to the specific environmental condition. Indeed, we verified growth support in shake flask experiments (Additional file 2). There are possibly additional false-negative results among the 134 substrates identified by the Biolog Phenotype Microarrays as non-growth supporting, if, simultaneously, the reference model iMT1026v3 would falsely lack the corresponding metabolic activities. Comparing our growth phenotypes with literature reports [9], we confirmed 17 out of 19 common substrates. As additional carbon sources, we identified raffinose and maltose (see Additional file 2).
Substrates with positive growth not predicted by simulations were added to the genome-scale reconstruction of O. polymorpha. As Table 2 shows, O. polymorpha grew on 35 substrates although simulations with iMT1026 predicted no growth. Of these previously unsuspected metabolized substrates, we added 27 substrates to the reconstruction iUL909. The remaining eight substrates were omitted because the annotation of metabolic pathways was unlikely. For example alanine amide has no associated pathway, whereas the degradation pathways for fucose and dulcitol involve galactose, which did not display growth in our test and was shown to be not metabolized by O. polymorpha [8]. We omitted gentiobiose because we only observed weak growth, although as a D-glucose disaccharide vigorous growth would be expected. O. polymorpha grew on L-leucine, and the model had all pathways required for metabolic activity, but a successful growth simulation was technically not achieved.

\section{Gene annotation and pathway correction}

New metabolic features were added and existing reactions corrected. All genes in the reconstruction iUL909 were checked in the methylotroph gene order

Table 2 Confusion matrix overview of the growth overlap between O. polymorpha in Biolog's Phenotype Microarray ${ }^{\mathrm{TM}}$ plates test and prediction according to the iMT1026v3 GSMM for K. phaffii 


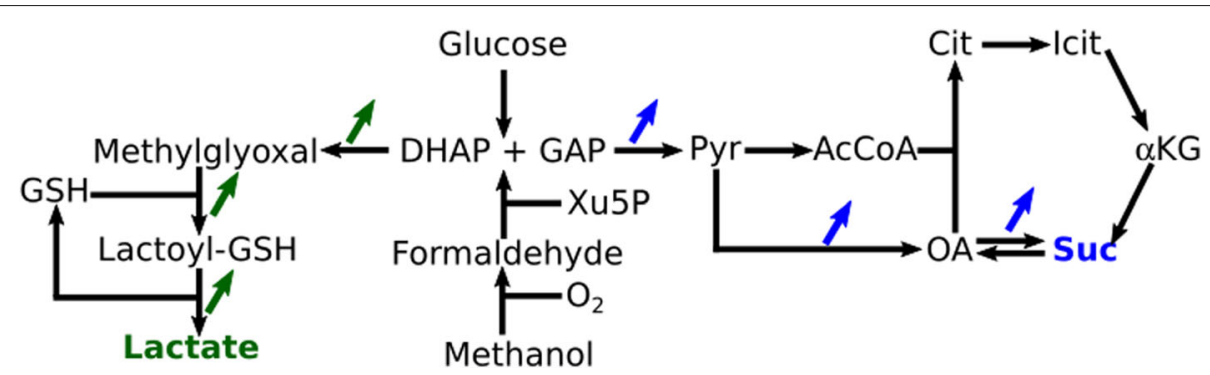

Fig. 3 Reaction activation candidates. Reactions to be activated according to FSEOF for overproduction of lactate (green) and succinate (blue). DHAP: Dihydroxyacetonephosphate, GAP: Glyceraldehydephosphate, GSH: Glutathione, Pyr: Pyruvate, AcCoA: Acetyl-CoA, Cit: Citrate, Icit: Isocitrate, $\alpha$ KG: $\alpha$ Ketoglutarate, Suc: Succinate, OA: Oxaloacetate

browser (MGOB) [23] and KEGG [24]. We accessed the UniProt TrEMBL [25] sequence source of $O$. polymorpha ${ }^{1}$ which listed 5167 proteins with only 422 EC number associated enzymes (Additional file 1). Due to the incomplete enzyme coverage by the UniProt annotation we relied on KEGG and the MGOB, and could functionally annotate $>97 \%$ of the genes. We identified enzymes within the biotin pathway converting 8-amino7oxononanoate to 7,8-diaminononoate while using $S$ adenosylmethionine as a co-substrate. To reconstitute the co-substrate we hypothesized that activity of $S$-adenosyl4-methylsulfanyl-2-oxobutanoate transaminase (EC number 2.6.1.12) would be present. We corrected the equation of over 30 reactions, for example fatty acid synthesis and uridine kinase reactions were corrected to maintain proton balance. There are still 59 unbalanced reactions due to multiple allowed charges in BIGG database and complex interrelations. Overall, the mass and charge balance is correct for more than $96 \%$ of all reactions as testified by the Memote report (see GitHub repository) and allows for faithful simulations.

The largest connected path we added was connected with the metabolism of erythritol. iUL909 contains four successive reactions that represent the further processing of erythritol. Erythritol is phosphorylated by a kinase to D-erythritol-1-phosphate, which in turn is converted to L-erythrulose-1-phosphate by means of a dehydrogenase and an epimerase. The L-erythrulose-1-phosphate is then split into dihydroxyacetone phosphate and formaldehyde. These cytosolic reactions were necessary to enable the observed growth on the sugar alcohol erythritol. The hydrolysis of many sugars with growth in the phenotype assays is catalyzed in our reconstruction by the maltase enzyme (EC-number 3.2.1.20). Maltase is known to display broad substrate specificity, which was also shown explicitly for O. polymorpha [26]. Two reactions were integrated which describe the activities of transketolase and transaldolase in the peroxisome of O. polymorpha. These

\footnotetext{
${ }^{1}$ accessed on $07 / 31 / 2018$
}

enzymes are essential for the alcoholic fermentation of xylose [27].

\section{Computational strain engineering tests}

Overproduction of important platform chemicals can be achieved with a limited number of genetic manipulations. We chose lactate and succinate as target compounds from methanol and glucose, and applied the FSEOF approach to identify reactions whose increased activity stimulates target production [28]. The optimized synthesis in iUL909 for lactate is routed via methylglyoxal generated from dihydroxyacetone phosphate [29]. Hence, stimulation of the glyoxalase system is predicted to enhance production (Fig. 3, green). Succinate production was increased in silico when the reactions of the lower glycolysis were more active, e.g., glyceraldehyde-3-phosphate dehydrogenase, pyruvate kinase, and pyruvate carboxylase (Fig. 3, blue). The strategies are similar for glucose and methanol. It is interesting to note that low uptake rates of methanol were more sensitive to reactions of the Xyl5P-pathway in the peroxisome. The strategy of reductive TCA cycle stimulation by anaplerotic reactions was experimentally shown to increase succinate production [30].

iUL909 fulfills key standards for reconstructed models and was tested with Memote and the SBML validator $[19,31]$. The SBML validator identifies a valid SBML Level 3v1 file, with flux balance constraints (FBC) in version 2 . The detected warnings relate to missing initial concentrations. The total Memote score is $45 \%$ (see Fig. 4, and the GitHub repository for the test result file). This is significantly improved with respect to the reference reconstruction for $K$. phaffii with a score of only $24 \%$.

\section{Conclusions}

A genome-scale metabolic model of $O$. polymorpha was constructed using homolog identification based on the existing GSMM of $K$. phaffii. The resulting GSMM iUL909 was further tailored for appropriate substrate utilization on the basis of phenotypic microarray and shake flask experiments. Although biomass composition and 


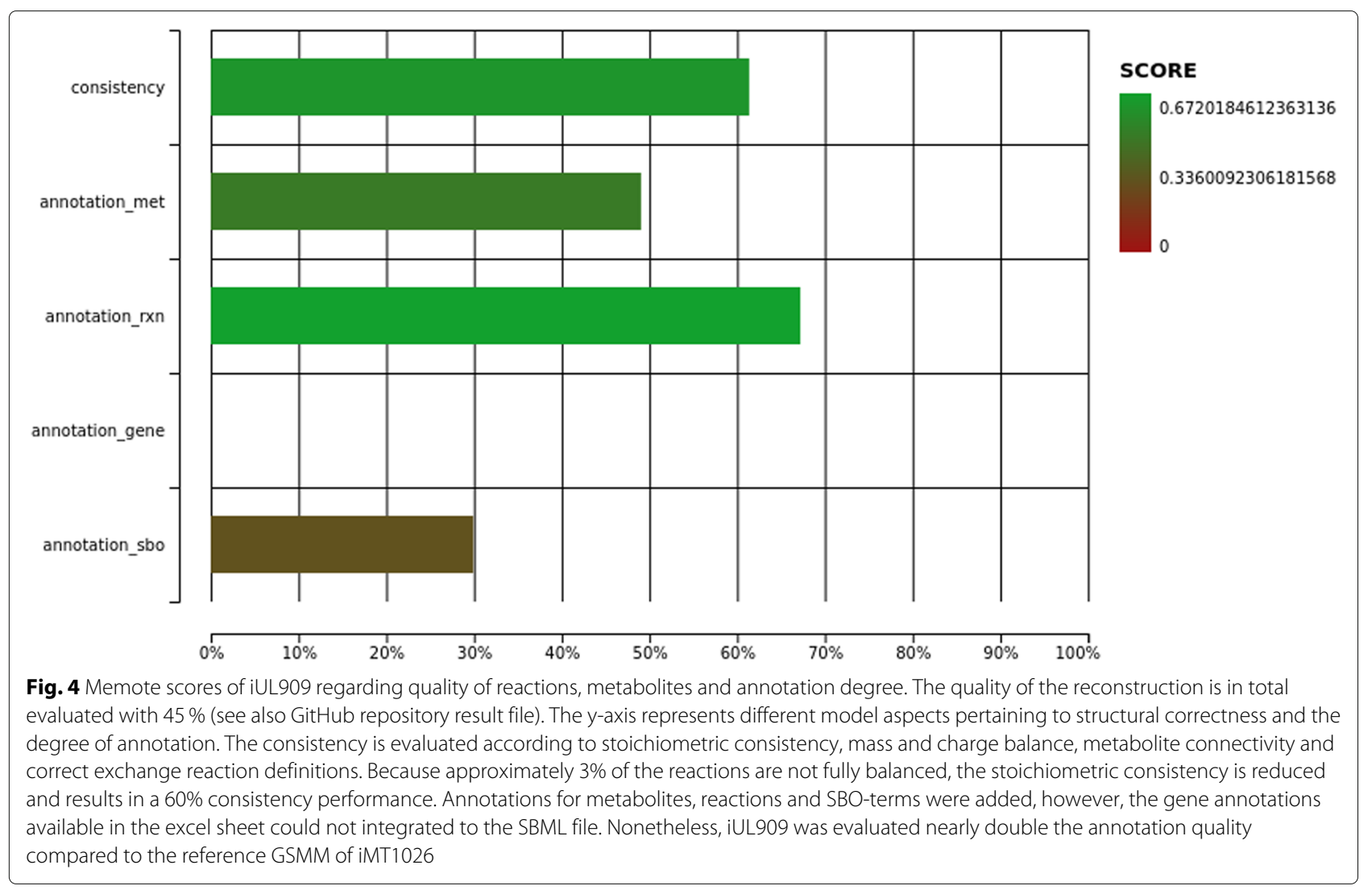

energetic parameters were adopted from $K$. phaffii, the resulting growth predictions were in good agreement with chemostat experiments for various industrially relevant substrates. The biotechnological applicability was explored by testing overproduction of lactate, succinate, showing that high productivity can be achieved with limited genetic manipulation. Thus, a metabolic model is now available for further strain engineering.

\section{Methods}

\section{Cultivation experiments}

The substrate utilization tests were conducted with the Biolog's Phenotype Microarray ${ }^{\mathrm{mi}}$ plates P1 and P2 with a total of 190 carbon sources. The medium was prepared with the manufacturer's inoculation medium IFY-0 and the dye mix $\mathrm{H}$ according to the guideline. The organism was cultivated in the microarrays in the Growth Profiler (EnzyScreen BV, Heemstede, Netherlands) at $37^{\circ} \mathrm{C}$ and $150 \mathrm{rpm}$, growth and respiratory activity was monitored by measuring the optical density (OD) at $490 \mathrm{~nm}$ for dye reduction and $750 \mathrm{~nm}$ for biomass in a plate reader (Synergy MX, BioTek Instruments Inc., USA) at $0 \mathrm{~h}$ and $72 \mathrm{~h}$.

To discriminate growth from non-growth, we considered the distribution of the OD increase for each plate at $490 \mathrm{~nm}$ and derived a suitable cut-off. The majo- rity of the OD increases clustered in a normal distribution at the lower OD-end, followed by a long tail with larger OD increases. The tail of the distribution characterizes explicit substrate respiration, while the normal distribution contains metabolized as well as non-utilized substrates. To identify metabolized substrates within the normal distribution, we first separated the normal distribution from the tail with the clear positive substrates. This was achieved by removing all values above the arithmetic mean within a plate. Then, a normal distribution was fitted over the remaining measurements, and one standard deviation above the mean was used as the cut-off for metabolized substrates (see Additional file 2). Moreover, growth was associated with a substrate only when at least $50 \%$ of replicates surpassed the threshold (duplicates for PM1, triplicates for PM2).

Separate-shake flask experiments were performed for the carbon substrates succinate, $\alpha$-ketoglutarate, $\alpha$ ketobutyrate, citrate, fumarate, L-malate and for the carbohydrates $\mathrm{D}$-xylose and D-ribose that failed to grow in the phenotype assays. The cultivation took place in CM2medium [30] and OD was tested after two, seven and thirteen days (see Additional file 2).

\section{Computational genome comparison}

The $O$. polymorpha genome sequence used for the 
model construction was based on [7] for strain NCYC 495 retrieved from Uniprot [25]. The $K$. phaffii GSMM iMT1026 [13] was used as reference model for the $O$. polymorpha reconstruction. Replacement of geneprotein-reaction (GPR) relationships was conducted by identification of homologs among $K$. phaffii and $O$. polymorpha. We used the genome sequence of $K$. phaffii GS115, the foundation of iMT1026, extracted from UniProt. The homologs were identified using the software package ProteinOrtho using the default parameter settings for homolog detection [32]. ProteinOrtho reports a single best homolog candidate for a $K$. phaffii input protein sequence. $K$. phaffii GPRs were replaced by the O. polymorpha homolog, while maintaining isoenzyme and multi-protein complex GPRs, which was possible for 908 GPRs. In the case of failed homolog mappings, manual Blast searches were conducted, and for the reactions of the central carbon metabolism manual comparison of gene annotations were performed with the JGI linked KEGG database of O. polymorpha [33] and the MGOB database [23].

The MGOB shows the local genomic organization among methylotrophic yeasts. $K$. phaffii MGOB-IDs of reactions in the central carbon metabolism were derived by querying the MGOB database with KEGG gene IDs. The MGOB-IDs were then used to locate the genes in their genomic context. The alignment with the homologs of all methylotrophic yeasts within MGOB provided information on annotation including subcellular localization. In this way, we could characterize 518 additional genes compared to UniProt. The model completeness and quality was tested with Memote [19]. SBML Level and Version tags were additionally validated with the SBML validator (http://sbml.org/Facilities/Validator).

\section{Flux balance analysis}

Simulations of the GSMM were performed with COBRAv3 on Matlab and with COBRApy. A Jupyter Notebook guide for the simulation of iUL909 and reproduction of experimental data as in Fig. 2 can be downloaded from GitHub (https://github.com/iAMBRWTH-Aachen/Opol-GSMM). Flux scanning based on enforced objective flux (FSEOF) [28] was performed in Matlab using the functions of COBRA toolbox to identify the targets for overexpression. Briefly, FSEOF was carried out in two stages. In the first stage, the maximum product flux (lactate or succinate) was computed by setting the biomass flux to zero and maximizing the objective function for product formation. In the second stage, the product flux was enforced in steps, and the biomass growth was set as an objective function and maximized. Reactions fluxes increasing monotonically represent targets for over-expression.

\section{Supplementary information}

Supplementary information accompanies this paper at

https://doi.org/10.1186/s12896-021-00675-w.

\section{Additional file 1: Supplementary information}

Additional file 2: Experimental data. Excel sheet with comparison of substrate utilization tests conducted in this paper and Suh et al. [9]. Detailed information on the results of Biolog's Phenotype Microarray ${ }^{\text {TM }}$ plates test, namely (i) a list of substrates with abiotic activity, (ii) for plate PM1: OD490/600 and selection of metabolized substrates, (iii) for plate PM1: OD490/600 and selection of metabolized substrates, (iv) shake flask growth results for the eight substrates not grown in phenotype assay (succinate, D-xylose, D-ribose, $\alpha$-ketoglutarate, $\alpha$-ketobutyrate, citrate, fumarate, malate).

Additional file 3: Genome scale metabolic model details. It contains a list of reactions with only one associated gene-protein-reaction association for which no homolog in O. polymorpha was identified. A list of all genes in iMT1026 for which no homologs were identified in O. polymorpha. Lists of new reactions and new genes added to the model. A table of literature values for growth rates and substrate uptake rates and finally the results of the FSEOF analysis.

\section{Abbreviations}

FSEOF: flux scanning based on enforced objective flux; GAM: growth associated maintenance; GPR: gene-protein-reaction relationship; GSMM: genome scale metabolic model; NGAM: non-growth associated maintenance; OD: optical density; SBML: systems biology markup language; TCA:

tricarboxylic acid cycle

\section{Acknowledgements}

We thank Frank Bergmann for support of the annotation of the SBML-file by supplying scripts. The authors are grateful to Ahmed Zahoor-ul-Hassan for stimulating discussions, Felix Küttner for experimental guidance, and Jan Engels for experimental support. Maria Bulimaga, Florencia Casanova, and Lisanne Jente developed and tested initial model versions.

\section{Authors' contributions}

UWL, LMB and BEE designed the original conception of the study. The biological experiments were performed by CVLS and SS. Simulations were performed by UWL, BAF, CVLS, and AR. Analysis of data was conducted by UWL, CVLS, SS, AR and BEE. The article was written by UWL, BAF, AR, SS, LMB, BEE. All authors have agreed to the final manuscript version.

\section{Funding}

This work was funded by the Biotechnology Research and Information Network AG (BRAIN AG) and by the German Federal Ministry of Education and Research (BMBF) as part of the Strategic Alliance Zero Carbon Footprint (Grant No. FKZ 031A217F), and by the Excellence Initiative of the German federal and state governments (G:(DE-82)EXS-PF-PFSDS015). The laboratory of L.M.B. is partially funded by the DFG (German Research Foundation) under Germany's Excellence Strategy - Exzellenzcluster 2186, 'The Fuel Science Center' ID: 390919832. BEE acknowledges support by the UQ-CSIRO Synthetic Biology Alliance. The funders had no influence on the design and publishing of the study. Open Access funding enabled and organized by Projekt DEAL.

\section{Availability of data and materials \\ O. polymorpha: NCYC 495 leu1.1 Genome Accession: AECK01000000 The genome scale model iUL909 generated during the current study is available from the corresponding author on reasonable request. A Jupyter Notebook for simulations, the Memote test result, and the reproduction of Fig. 1 is available on GitHub: https://github.com/iAMB-RWTH-Aachen/Opol-GSMM}

\section{Ethics approval and consent to participate}

Not applicable

\section{Consent for publication \\ Not applicable}




\section{Competing interests}

The authors declare that they have no competing interests.

\section{Author details}

${ }^{1}$ Institute of Applied Microbiology-iAMB, Aachen Biology and Biotechnology-ABBt, RWTH Aachen University, Worringer Weg 1, 52074 Aachen, Germany. ${ }^{2}$ Genome Institute of Singapore, 60 Biopolis Street, Genome, 03-01, 138672 Singapore, Singapore. ${ }^{3}$ Australian Institute for Bioengineering and Nanotechnology, The University of Queensland, QLD 4072 Brisbane, Australia. ${ }^{4}$ CSIRO Future Science Platform in Synthetic Biology, Commonwealth Scientific and Industrial Research Organisation (CSIRO), ACT 2601, Black Mountain, Australia.

\section{Received: 28 August 2020 Accepted: 4 December 2020} Published online: 15 March 2021

\section{References}

1. Ramezani-Rad M, Hollenberg CP, Lauber J, Wedler H, Griess E, Wagner C, Albermann K, Hani J, Piontek M, Dahlems U. The Hansenula polymorpha (strain CBS4732) genome sequencing and analysis. FEMS Yeast Res. 2003;4(2):207-15. https://doi.org/10.1016/S1567-1356(03)00125-9.

2. Iguchi $H$, Yurimoto $H$, Sakai Y. Interactions of methylotrophs with plants and other heterotrophic bacteria. Microorganisms. 2015;3(2):137-51. https://doi.org/10.3390/microorganisms3020137.

3. Kunze G, Kang HA, Gellissen G. \emph\{Hansenula polymorpha (Pichia angusta)\}: Biology and Applications. Yeast Biotechnol Divers Appl. 200947-64. https://doi.org/10.1007/978-1-4020-8292-4-3.

4. Scheidle M, Jeude M, Dittrich B, Denter S, Kensy F, Suckow M, Klee D, Büchs J. High-throughput screening of \emph\{Hansenula polymorpha\} clones in the batch compared with the controlled-release fed-batch mode on a small scale. FEMS Yeast Res. 2010;10(1):83-92. https://doi.org/ 10.1111/j.1567-1364.2009.00586.x.

5. Lehnen M, Ebert BE, Blank LM. A comprehensive evaluation of constraining amino acid biosynthesis in compartmented models for metabolic flux analysis. Metab Eng Comm. 2017;5:34-44. https://doi.org/ 10.1016/j.meteno.2017.07.001

6. Liebal UW, Blank LM, Ebert BE. CO2 to succinic acid - Estimating the potential of biocatalytic routes. Metab Eng Commun. 2018;7:. https://doi. org/10.1016/j.mec.2018.e00075.

7. Riley R, Haridas S, Wolfe KH, Lopes MR, Hittinger CT, Göker M, Salamov AA, Wisecaver JH, Long TM, Calvey CH, al E. Comparative genomics of biotechnologically important yeasts. Proc Natl Acad Sci U S A. 2016;113(35):9882-7. https://doi.org/10.1073/pnas.1603941113.

8. Kurtzman CP, Robnett CJ. Systematics of methanol assimilating yeasts and neighboring taxa from multigene sequence analysis and the proposal of \emph\{Peterozyma gen. nov.\}, a new member of the lemph\{Saccharomycetales\}. FEMS Yeast Res. 2010;10(3):353-61. https:// doi.org/10.1111/j.1567-1364.2010.00625.x.

9. Suh S-O, Zhou JJ. Methylotrophic yeasts near \emph\{Ogataea (Hansenula) polymorpha\}: a proposal of \emph\{Ogataea angusta comb. nov.\} and \emph\{Candida parapolymorpha sp. nov.\}. FEMS Yeast Res. 2010;10(5):631-8. https://doi.org/10.1111/j.1567-1364.2010.00634.x.

10. Ravin NV, Eldarov MA, Kadnikov W, Beletsky AV, Schneider J, Mardanova ES, Smekalova EM, Zvereva MI, Dontsova OA, Mardanov AV, al E. Genome sequence and analysis of methylotrophic yeast lemph\{Hansenula polymorpha\} \{DL1\}. BMC Genomics. 2013;14(1):837. https://doi.org/10.1186/1471-2164-14-837

11. Theron CW, Berrios J, Delvigne F, Fickers P. Integrating metabolic modeling and population heterogeneity analysis into optimizing recombinant protein production by \emph\{Komagataella (Pichia) pastoris\}. Appl Microbiol Biotechnol. 2018;102(1):63-80. https://doi.org/ 10.1007/s00253-017-8612-y

12. Peña DA, Gasser B, Zanghellini J, Steiger MG, Mattanovich D. Metabolic engineering of Pichia pastoris. Metab Eng. 2018;50:2-15. https://doi.org/ 10.1016/j.ymben.2018.04.017.

13. Tomàs-Gamisans $M$, Ferrer P, Albiol J. Fine-tuning the P. pastoris iMT1026 genome-scale metabolic model for improved prediction of growth on methanol or glycerol as sole carbon sources. Microb Biotechnol. 2018;11(1):224-37. https://doi.org/10.1111/1751-7915.12871.

14. Ye R, Huang M, Lu H, Qian J, Lin W, Chu J, Zhuang Y, Zhang S. Comprehensive reconstruction and evaluation of Pichia pastoris genome-scale metabolic model that accounts for 1243 ORFs. Bioresour Bioprocess. 2017;4(1):22. https://doi.org/10.1186/s40643-017-0152-x.
15. Cabeç-Silva C, Madeira-Lopes A. Temperature relations of yield, growth and thermal death in the yeast Hansenula polymorpha. Z Allg Mikrobiol. 1984;24(2):129-32. https://doi.org/10.1002/jobm.19840240216.

16. Ryabova O, Chmil O, Sibirny A. Xylose and cellobiose fermentation to ethanol by the thermotolerant methylotrophic yeast. FEMS Yeast Res. 2003;4(2):157-64. https://doi.org/10.1016/S1567-1356(03)00146-6.

17. Siverio JM. Assimilation of nitrate by yeasts. FEMS Microbiol Rev. 2002;26(3):277-84. https://doi.org/10.1111/j.1574-6976.2002.tb00615.x.

18. Gellissen G, Kunze G, Gaillardin C, Cregg J, Berardi E, Veenhuis M, VanderKlei I. New yeast expression platforms based on methylotrophic and and on dimorphic and - A comparison. FEMS Yeast Res. 2005;5(11): 1079-96. https://doi.org/10.1016/j.femsyr.2005.06.004.

19. Lieven C, Beber ME, Olivier BG, Bergmann FT, Ataman M, Babaei P, Bartell JA, Blank LM, Chauhan S, Correia K, et. al. MEMOTE for standardized genome-scale metabolic model testing. Nat Biotechnol. 2020;38(3):272-6. https://doi.org/10.1038/s41587-020-0446-y.

20. Van Dijken LP, Otto R, Harder W. Growth of \emph\{Hansenula polymorpha\} in a methanol-limited chemostat. Arch Microbiol. 1976;111(1-2):137-44. https://doi.org/10.1007/bf00446560.

21. De Koning W, Harder W, Dijkhuizen L. Glycerol metabolism in the methylotrophic yeast \emph\{Hansenula polymorpha\}: phosphorylation as the initial step. Arch Microbiol. 1987;148(4):314-20. https://doi.org/10. 1007/BF00456710.

22. Moon H, Kim SW, Lee J, Rhee SK, Choi ES, Kang HA, Kim IH, Hong SI. Independent exponential feeding of glycerol and methanol for fed-batch culture of recombinant \emph\{Hansenula polymorpha\} DL-1. Appl Biochem Biotechnol. 2003;111(2):65-79. https://doi.org/10.1385/ABAB: 111:2:65.

23. Douglass AP, Byrne KP, Wolfe KH. The Methylotroph Gene Order Browser (MGOB) reveals conserved synteny and ancestral centromere locations in the yeast family Pichiaceae. FEMS Yeast Res. 2019;19(6):058. https://doi. org/10.1093/femsyr/foz058.

24. Kanehisa M, Goto S. KEGG: kyoto encyclopedia of genes and genomes. Nucleic Acids Res. 2000;28(1):27-30. https://doi.org/10.1093/nar/28.1.27.

25. UniProt Consortium. UniProt: a worldwide hub of protein knowledge. Nucleic Acids Res. 2019;47(D1):506-15. https://doi.org/10.1093/nar/ gky1049.

26. Viigand K, Visnapuu T, Mardo K, Aasamets A, Alamäe T. Maltase protein of \emph\{Ogataea (Hansenula) polymorpha\} is a counterpart to the resurrected ancestor protein ancMALS of yeast maltases and isomaltases. Yeast. 2016;33(8):415-32. https://doi.org/10.1002/yea.3157.

27. Kurylenko OO, Ruchala J, Vasylyshyn RV, Stasyk OV, Dmytruk OV, Dmytruk KV, Sibirny AA. Peroxisomes and peroxisomal transketolase and transaldolase enzymes are essential for xylose alcoholic fermentation by the methylotrophic thermotolerant yeast, Ogataea (Hansenula) polymorpha. Biotechnol Biofuels. 2018;11(1):197. https://doi.org/10.1186/ s13068-018-1203-z.

28. Choi HS, Lee SY, Kim TY, Woo HM. In silico identification of gene amplification targets for improvement of lycopene production. Appl Env Microbiol. 2010;76(10):3097-105. https://doi.org/10.1128/AEM.00115-10.

29. Inoue $Y$, Kimura A. Methylglyoxal and regulation of its metabolism in microorganisms. Adv Microb Physiol. 1995;37:177-227. https://doi.org/10. 1016/S0065-2911(08)60146-0.

30. Yan D, Wang C, Zhou J, Liu Y, Yang M, Xing J. Construction of reductive pathway in \emph\{Saccharomyces cerevisiae\} for effective succinic acid fermentation at low pH value. Bioresour Technol. 2014;156:232-9. https:// doi.org/10.1016/j.biortech.2014.01.053.

31. Bornstein BJ, Keating SM, Jouraku A, Hucka M. LibSBML: an API library for SBML. Bioinforma. 2008;24(6):880-1. https://doi.org/10.1093/ bioinformatics/btn051.

32. Lechner M, Findeiß S, Steiner L, Marz M, Stadler PF, Prohaska SJ. Proteinortho: detection of (co-) orthologs in large-scale analysis. BMC Bioinforma. 2011;12(1):124. https://doi.org/10.1186/1471-2105-12-124.

33. Grigoriev IV, Nikitin R, Haridas S, Kuo A, Ohm R, Otillar R, Riley R, Salamov A, Zhao X, Korzeniewski F, Smirnova T, Nordberg H, Dubchak I, Shabalov I. MycoCosm portal: Gearing up for 1000 fungal genomes. Nucleic Acids Res. 2014;42(D1):699-704. https://doi.org/10.1093/nar/ gkt1183.

\section{Publisher's Note}

Springer Nature remains neutral with regard to jurisdictional claims in published maps and institutional affiliations. 